American Journal of Applied Sciences 5 (11): 1566-1571, 2008

ISSN 1546-9239

(C) 2008 Science Publications

\title{
The Numerical Estimation of Thermal Contact Resistance in Contacting Surfaces
}

\author{
${ }^{1}$ M.H. Shojaefard and ${ }^{2}$ K. Goudarzi \\ ${ }^{1}$ Department of Automotive Engineering, \\ ${ }^{2}$ Department of Mechanical Engineering, \\ Iran University of Science and Technology, Tehran, Iran
}

\begin{abstract}
For some manufacturing processes, the heat transfer between the components, the tools and the environment has an effect on tool-life and the accuracy of the formed component. Consequently, the measurement of Thermal Contact Resistance (TCR) is of increasing interest to researchers and industrial engineers participating in the manufacture of high-precision components. A new transient method and measurement apparatus are used in which the measurements are conducted on specimens, which are retained under pressure. An apparent advantage of this method is the ability to estimate the TCR under specifically controlled conditions. The other advantage is that no prior information is needed on the variation of the TCR, since the solution automatically determines the functional form over the domain specified. Therefore, in this research, a new method of determining TCR has been successfully used to measure the dependence of TCR on the pressure and the specimen texture.
\end{abstract}

Key words: Heat transfer, thermal contact conductance, inverse problem

\section{INTRODUCTION}

The energy expended to plastically deform materials in manufacturing processes such as metalforming processes is converted almost entirely into heat. This energy increases the temperature of the formed component and the tools while some of it is dissipated to the environment. The heat transfer to the component and tools has an effect on the accuracy of the formed component. Consequently, heat transfer from the work-material to the tool and the environment is of increasing interest to researchers and engineers participating in the manufacturing of high-precision components.

As a result of manufacturing processes, real surfaces have roughness and surface curvature. The real contact occurs only over microscopic contacts, which are typically only a few percent of the apparent contact area. Because of the surface curvature of contacting bodies, the macro-contact area is formed, the area where micro-contacts are distributed randomly. The heat flow must pass through the macro-contact and then micro-contacts to transfer from one surface to another. This phenomenon leads to a relatively high temperature drop across the interface. Also, the thermal contact resistance is a complex interdisciplinary problem, which includes geometrical, mechanical and thermal analyses. Analytical, experimental and numerical models have been developed to predict TCR since the 1930's. These models are applicable only to the limiting cases and none of them covers the general nonconforming rough contact ${ }^{[1]}$.

The reliability of analytical approaches depends on the accuracy of the material properties and the physical parameters that influence heat transfer between surfaces. The last refers to values for specific heat, thermal contact resistance and coefficient of thermal expansion of both the component and the tool materials; whilst a further consideration is the allocation of a value for thermal contact resistance, since this determines the thermal balance in the component/tool/ environment system ${ }^{[1]}$.

Thermal contact resistance, $R_{c}$, is defined as follows ${ }^{[2]}$ :

$$
\mathrm{R}_{\mathrm{C}}=\frac{\Delta \mathrm{T}}{\mathrm{q}}
$$

where, $\Delta \mathrm{T}$ is the temperature difference at the contacting surfaces and $\mathrm{q}$, heat flux, defined as:

$$
q=\frac{d}{d A}\left(\frac{d Q}{d t}\right)
$$

Corresponding Author: K. Goudarzi, Department of Mechanical Engineering, Iran University of Science and Technology Narmak, 16844, Tehran, Iran Tel: ++98 2173913963 Fax: ++98 2177491225 
It is recognized that thermal contact resistance is a function of several parameters, the dominant ones being the type of contacting materials, the macro-and microgeometry of the contacting surfaces, the temperature, the interfacial pressure, the type of lubricant or contaminant and its thickness. Further, the variation of the interfacial pressure with time has a significant influence on the thermal contact resistance. Until now, several different thermodynamic models have been used to compute the thermal contact resistance ${ }^{[3,4]}$.

Measurements of the thermal contact resistance have been carried out while heat transfer was either in steady-state or transient condition experiments were conducted using devices that contained two specimens or two tools with a specimen sandwiched between them. These experiments were followed by an assessment of the thermal contact resistance while the test specimen was deformed plastically; a further development involved the integration of thermocouples in the specimen. In the simplest case of steady-state heat transfer, the thermal contact resistance may be determined using Eq. 1. The thermal contact resistance is assumed to be the value that provides the best match between simulation and experimental results. A new method is used based on the solution of an inverse problem. The sequential inverse method has been used to determine the thermal contact resistance in metal forming processes. Review of publication ${ }^{[5-12]}$ suggests that values of thermal contact resistance vary significantly, perhaps due to the fact that these were derived using different experimental approaches. Published results were derived from experiments of different configurations, such as different materials, surface preparation, pressure and temperature, thus disabling comparison. These tests were conducted using specimens similar to those used in previous research ${ }^{[5]}$. Further, the variation of thermal contact resistance at pressures is shown trends which appeared to depend on the work material and experimental conditions.

It may be concluded that experiments used to determine thermal contact resistance include some errors. Results depend on the measuring devices used in the experiments and on the method of processing the experimental data.

Therefore, the objective of this study is to develop an analytical model for predicting TCR for the entire range of non-conforming contacts. Therefore, a new approach for deriving values of thermal contact resistance under differing interfacial conditions is presented, together with results on the dependence of TCR on pressure and contact type (similar or dissimilar contact). A clear advantage of this method is the ability to measure the thermal contact resistance under precisely controlled and continuously sustained conditions. The other advantage of the present method is that no a priori information is needed on the variation of the unknown quantities, since the solution automatically determines the functional form over the domain specified.

\section{EQUIPMENT AND PROCEDURE}

Test equipment: The proposed approach is based on transient heat flow along two cylindrical tools and through a specimen. The distinct characteristic of this approach is using a thin specimen of the same diameter as the tool. The experimental equipment is shown schematically in Fig. 1. The lower part of the device is attached to the platen while the upper is attached to the ram. The upper specimen is equipped with a heater while the lower specimen is fitted with a heat-sink (reservoir). The test specimens are located on the setup.

A compressive load was exerted on the specimens by a simple mechanical system, including a free disk and several dead weights. The temperatures of the specimens are controlled by using circulating cooling water at the bottom specimen and a heater overhead the top specimen. The steel plate base is applied to hold the cooling system. The K-Type thermocouples were used for all of the temperature measurements. The thermocouples were mounted in holes drilled perpendicular to the axis of symmetry of the specimens. All the thermocouples were connected to a Data Acquisition system through the feedthroughs. The data acquisition set-up was composed of 8 channels and interfaced to an Intel PC/Pentium II computer. The Data Acquisition system included a SCSI board and MATLAB software, used for collecting data, storing data and data analysis.

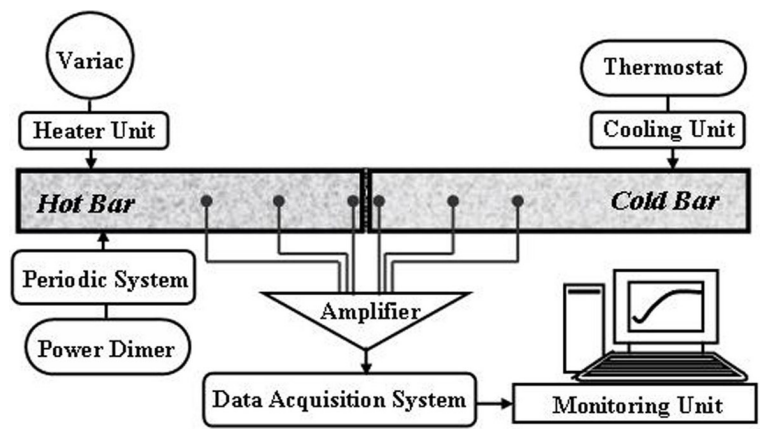

Fig. 1: Schematic of experimental setup 
Am. J. Applied Sci., 5 (11): 1566-1571, 2008

Table 1: Thermo-physical properties of specimens at $300 \mathrm{~K}$

\begin{tabular}{lll} 
& Aluminum & Stainless steel \\
\hline Conductivity $(\mathrm{W} / \mathrm{mK})$ & 177 & 37.7 \\
Heat capacitance $(\mathrm{j} / \mathrm{kg} . \mathrm{K})$ & 875 & 444 \\
Density $\left(\mathrm{kg} / \mathrm{m}^{3}\right)$ & 2770 & 7822 \\
\hline
\end{tabular}

Specimens specifications: Cylindrical specimens (8 $\mathrm{mm}$ diameter and $20 \mathrm{~cm}$ lenght) were made from stainless steel and aluminum. Contact surfaces of all specimens were grounded before test using machining. Two sets of specimens, including similar and dissimilar contact, were used in this research. Thermo-physical properties of specimens are shown in Table 1 at $300 \mathrm{~K}$. It should be noted that with increasing the temperature to $800 \mathrm{~K}$, these properties do not vary significantly. Thus, properties of specimens at $300 \mathrm{~K}$ were used for calculations.

Test procedure: The test specimens were located accurately on the setup. The heat flux settings of the main heater and the temperature of the cooling water were used to define the thermal conditions of the experiments. The continuous changes in temperature along the specimens were monitored until a steady-state condition was achieved. At the point that the temperature distributions along the specimens were stable and the difference of heat fluxes in both specimens was within a specified limit, readings from the thermocouples were used to compute the thermal contact resistance. Also, all experiments were conducted with dry interfaces.

Computational considerations: As it has been already mentioned, heat flow in contact surface was achieved by applying a heat source to the upper specimen and a heat sink to the lower specimen. The temperature distribution along the specimens, measured along their axis by $3 \mathrm{~K}$-Type thermocouples in each specimen, was used to calculate the heat flux and temperature difference at the interface Fig. 2. It could be assumed that the temperature drop on both contact surfaces of the specimen was defined by:

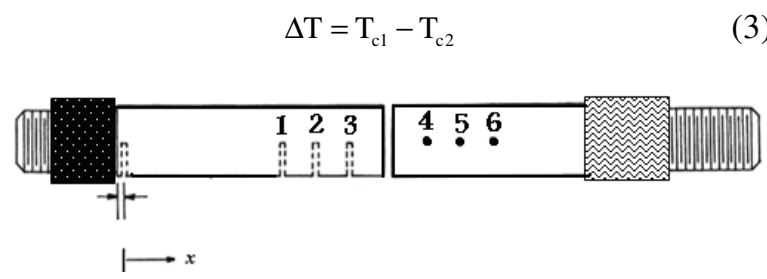

Fig. 2: The test specimens
Thus, using Eq. 1, the thermal contact resistance may be determined using the following equation:

$$
\mathrm{R}_{\mathrm{C}}=\left(\mathrm{T}_{\mathrm{c} 1}-\mathrm{T}_{\mathrm{c} 2}\right) / \mathrm{q}
$$

where, $\mathrm{T}_{\mathrm{c} 1}$ and $\mathrm{T}_{\mathrm{c} 2}$ are the temperature of contact surfaces of specimens and are estimated by the leastsquares method.

\section{RESULTS AND DISCUSSION}

Experimental results: Three $8 \mathrm{~mm}$ diameter specimens of $20 \mathrm{~cm}$ height with thermo-mechanical properties of stainless steel and aluminum were used in simulations. Friction between the specimens was assumed to be zero to eliminate heat generation due to friction. The lateral surfaces of specimens diffused heat by convection to the ambient. Therefore, the lateral surfaces of the specimens are insulated with glass wool as an insulator. The top surface of the upper specimen was retained at a constant heat flux and the bottom surface of the lower specimen was retained at a constant temperature of $20.0^{\circ} \mathrm{C}$.

Temperature variations with time at near the contact surfaces for similar contact (steel-steel) were shown in Fig. 3. Also, Fig. 4 shows the temperature variations with time whole specimens.

The values of thermal contact resistance were computed using Eq. 4. The thermal contact resistance is a function of many parameters such as pressure, contact type and surface roughness. For the measured values of surface texture, the variation of thermal contact resistance with the applied pressure is shown in Fig. 5.

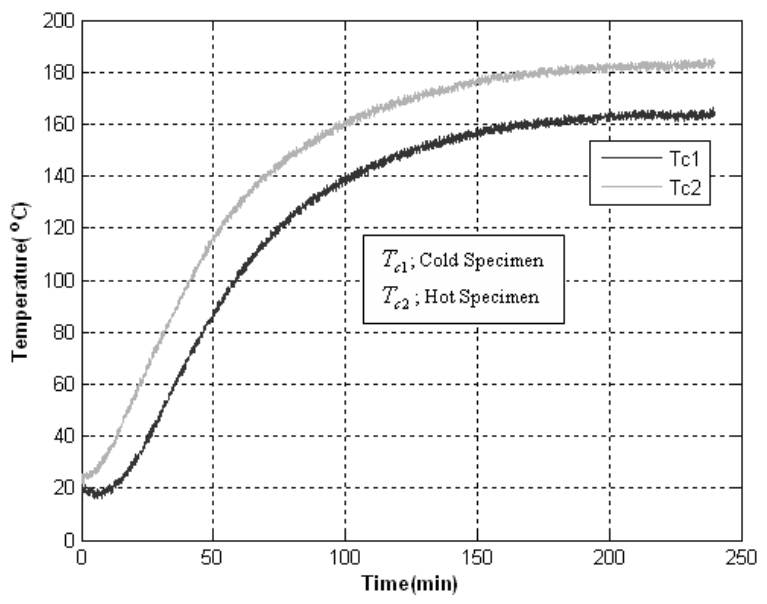

Fig. 3: Variation of temperature with time at near the contact surfaces; similar contact 


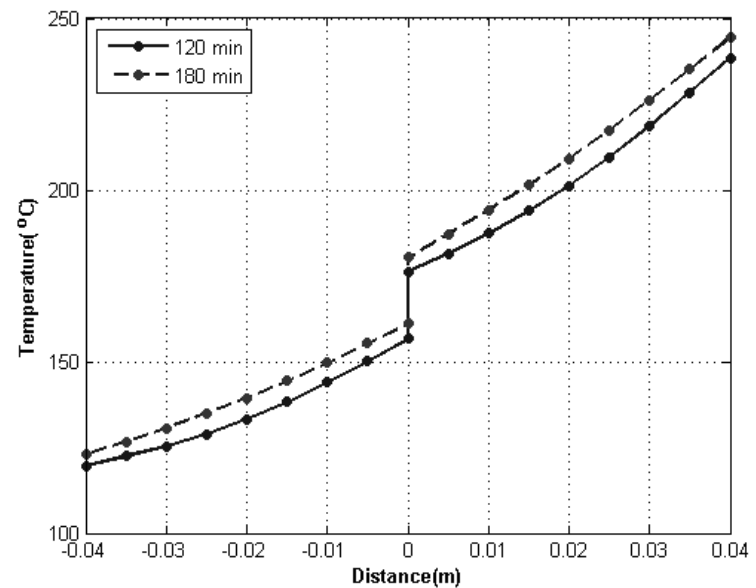

Fig. 4: Variation of temperature with time across specimens

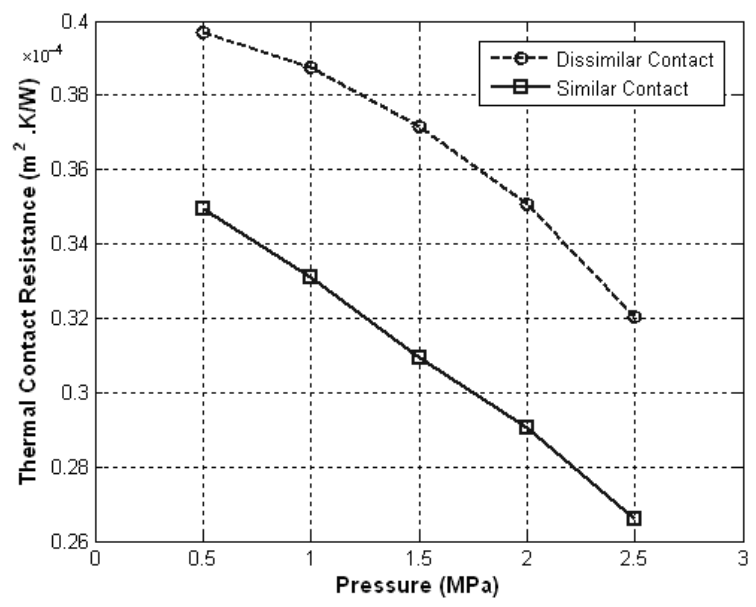

Fig. 5: Variation of thermal contact resistance with contact pressure (experimental method)

Thermal contact resistance bears an exponential relationship to interfacial pressure. This result agrees with the paper ${ }^{[5]}$ for experiments with aluminum specimens under dry conditions. Further, it was found that the magnitude of the thermal contact resistance increased more rapidly with pressure for a dissimilar contact (stainless steel and aluminum).

\section{INVERSE PROBLEM}

Direct Problem: The geometry for the onedimensional physical problem considered here is shown in Fig. 2. Two specimens of equal lengths are contacting with a contact conductance $R_{c}(t)$ at the interface. The mathematical formulation of this problem is given in dimensionless form as:
Specimen $1(0 \leq x \leq 1)$ :

$\frac{\partial^{2} \mathrm{~T}_{1}}{\partial \mathrm{x}^{2}}=\frac{\partial \mathrm{T}_{1}}{\partial \mathrm{t}}$ in $0<\mathrm{x}<1$ for $\mathrm{t}>05-\mathrm{a}$

$\mathrm{T}_{1}=0$ at $\mathrm{x}=0$ for $\mathrm{t}>05-\mathrm{b}$

$-\frac{\partial \mathrm{T}_{1}}{\partial \mathrm{x}}=\left[\mathrm{T}_{1}-\mathrm{T}_{2}\right] / \mathrm{R}_{\mathrm{C}}$ at $\mathrm{x}=1$ for $\mathrm{t}>05-\mathrm{c}$

$\mathrm{T}_{1}(\mathrm{x}, 0)=\mathrm{T}_{\mathrm{inf}} \quad 5-\mathrm{d}$

Specimen $2(1 \leq \mathrm{x} \leq 1+\mathrm{L})$ :

$\frac{\partial^{2} \mathrm{~T}_{2}}{\partial \mathrm{x}^{2}}=\frac{1}{\alpha} \frac{\partial \mathrm{T}_{2}}{\partial \mathrm{t}}$ in $1<\mathrm{x}<1+\mathrm{L}$ for $\mathrm{t}>06-\mathrm{a}$

$-k \frac{\partial T_{2}}{\partial x}=\left[T_{1}-T_{2}\right] / R_{C}$ at $x=1$ for $\mathrm{t}>06-b$

$\mathrm{T}_{2}=1$ at $\mathrm{x}=1+\mathrm{L}$ for $\mathrm{t}>06-\mathrm{c}$

$\mathrm{T}_{2}(\mathrm{x}, 0)=\mathrm{T}_{\mathrm{inf}} \quad 6-\mathrm{d}$

The direct problem under consideration was concerned with the determination of the temperature field in the specimens when the thermo-physical properties, interface thermal contact resistance and the boundary conditions at the outer ends of the specimens are known.

Solution procedure of inverse problem: It is assumed that no prior information is available on the functional form of $R_{c}(t)$ is known. We are following the function $R_{c}(t)$ over the whole time domain, with the assumption that $R_{c}(t)$ belongs to the Hilbert space of squareintegrable functions in the time domain ${ }^{[13]}$.

The solution of the present inverse problem is to be obtained in such a way that the following function is minimized:

$S\left[R_{C}(t)\right]=\int_{t=0}^{\tau}\left[\sum_{j=1}^{N_{1}}\left(T_{1 j}-Y_{1 j}\right)^{2}\right] d t+\int_{t=0}^{\tau}\left[\sum_{k=1}^{N_{2}}\left(T_{2 k}-Y_{2 k}\right)^{2}\right] d t(7)$

where, $T_{1 j}$ and $T_{2 k}$ are the estimated temperatures at the measurement locations in specimens and $Y_{1 j}$ and $Y_{2 k}$ are the measured temperatures.

The Conjugate Gradient Method (CGM) by utilizing the ideas based on perturbation principles; transform the inverse problem to solution of three 


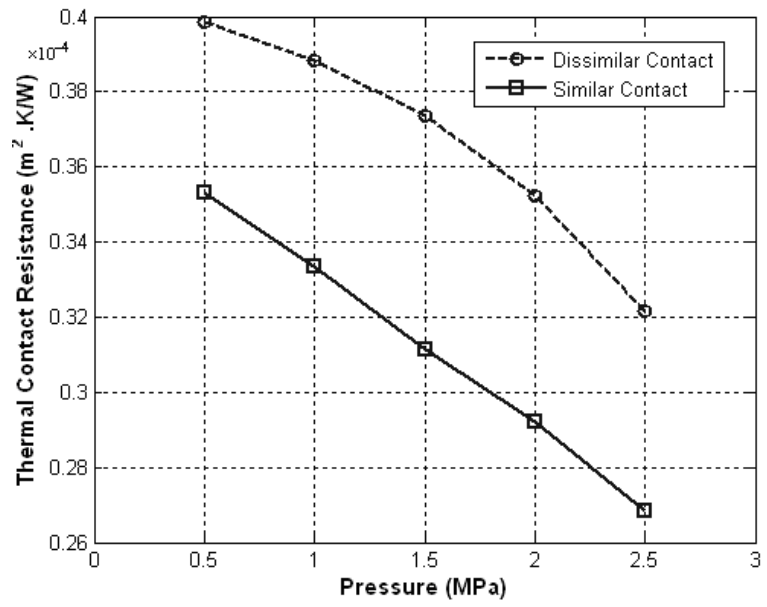

Fig. 6: Variation of thermal contact resistance with contact pressure (CGM method)

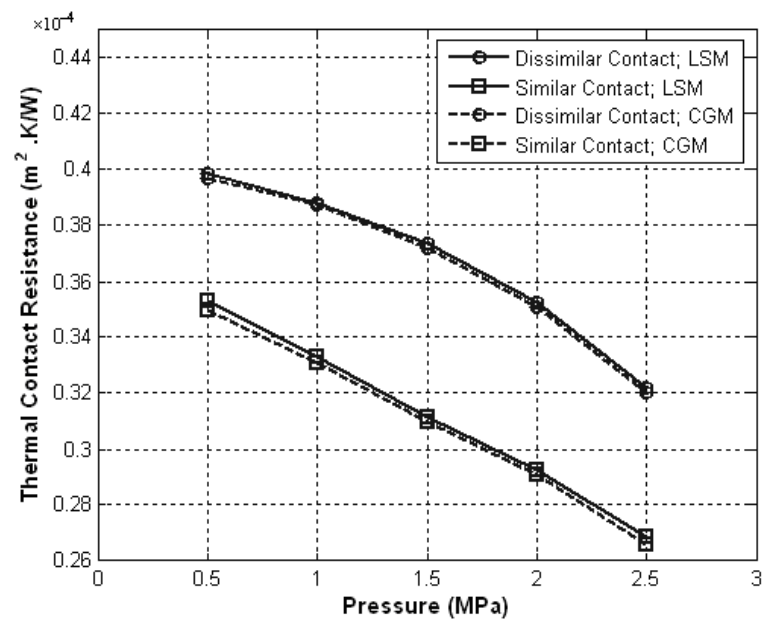

Fig. 7: Variation of thermal contact resistance with contact pressure

simple problems called the direct problem, the sensitivity problem and the adjoint problem together with the gradient equation. The advantage of the present method is that no a priori information is needed on the variation of the unknown quantities, since the solution automatically determines the functional form over the domain specified ${ }^{[14]}$.

Result applying exact data: Fig. 6 shows the results obtained with exact measurements for the varying TCR. Note that for the case considered, the results obtained with the CGM are exactly the same as the TCR function supposed by Eq. 4 (Fig. 7). It should be noted that, the solutions with the CGM are very stable and do not exhibit oscillations.

\section{CONCLUSION}

The conducted research concludes the following results:

- The thermal contact resistance is extremely difficult to measure. Thus, a new method of determining thermal contact resistance has been successfully used to estimate the dependence of thermal contact resistance on pressure and surface texture

- Thermal contact resistance decreases with pressure and increases with the surface roughness of the contact surfaces.

- Decrease of the value of thermal contact resistance with pressure was more pronounced for specimens with smooth surface finishes

- The influence of the pressure-dependent thermal contact resistance on the specimen surface temperatures would be more prominent in processes where high interface pressures occur

- The obtained results with the CGM are very stable and do not exhibit oscillations

\section{NOMENCLATURE}

\section{Notation:}

A:

$\mathrm{k}:$

$\mathrm{N}$ :

Q:

q:

$\mathrm{R}_{\mathrm{c}}$ :

$\mathrm{S}[\mathrm{]}$ :

$\mathrm{T}$ :

$\mathrm{t}:$

$\mathrm{X}$ :

Y:

A/D:

Subscript

1,2:

$1 \mathrm{j}:$

$2 \mathrm{k}:$

c:

inf:

\author{
Area \\ Thermal conductivity \\ Number of sensors \\ Heat transfer \\ Heat flux \\ Thermal contact resistance \\ Loss function \\ Estimated temperature \\ Time \\ Distance \\ Measured temperature \\ Analog to digital \\ Specimen 1,2 \\ Specimen 1 \\ Specimen 2 \\ Contact \\ Ambient
}

\section{REFERENCES}

1. Shen, G., S.L. Semiatin, E. Kropp and T. Altan, 1992. A technique to compensate for temperature history effect in the simulation of non-isothermal forging processes. J. Mater. Process Technol., 33 (1-2): 125-140. 
2. Madhusudana, C.V., 1996. Thermal Contact Conductance. Springer, Berlin, ISBN 0-38794534-2.

3. Degiovanni, A., A.S. Lamine and C.H. Moyne, 1992. Thermal contact in transient state-a new model and two experiments. J. Thermophys. Heat Transfer. 6 (2): 356-363.

4. Jurkowski, T., Y. Jarny and D. Delaunay, 1992. Simultaneous identification of thermal conductivity and thermal contact resistance without internal temperature measurements. Proceedings of the 3rd UK Conference on Heat Transfer, Birmingham.

5. Jain, V.K., 1990. Determination of heat transfer coefficient for forging application. J. Mater. Shap. Technol., 8 (3): 193-202.

6. Semiatin, S.L., E.W. Collings, V.E. Wood and T. Altan, 1987. Determination of the interface heat transfer coefficient for non-isothermal bulk forming processes. ASME J. Eng. Ind., 109: 49-57.

7. Malinowski, Z., J.G. Lenard and M.E. Davies, 1994. A study of the heat transfer coefficient as a function of temperature and pressure. J. Mater. Process Technol., 41: 125-142.

8. Nshama, W. and J. Jeswiet, 1995. Evaluation of temperature and heat transfer conditions at the metal-forming interface. CIRP AnnalsManufacturing Technol., 44 (1): 201-204.
9. Burte, P.R., I.M. Yong-Taek, T. Altan and S.L. Semiatin, 1990. Measurements and analysis of the heat transfer and friction during hot forging. J. Eng. Ind., 112: 332-339.

10. Dadras, P. and W.R. Wells, 1984. Heat transfer aspects of nonisothermal axisymmetric upset forging. J. Eng. Ind., 106: 187-195.

11. Goizet, V., B. Bourouga and J.P. Bardon, 1998. Experimental study of the thermal boundary conditions at the workpiece-die interface during hot forging. Proceedings of the 11th IHTC, Korea, August.

12. Jewiet, J., W. Nshama and P.H. Oasthuizen, 1998. Evaluation of temperature and heat transfer conditions in metal forming. Proceedings of the 11th International Heat Transfer Conference, Kyongju, South Korea.

13. Alifanov, O.M, 1994. Inverse Heat Transfer Problems. Springer-Verlag, New York.

14. Ozisik, M.N. and H.R.B. Orlande, 2000. Inverse Heat Transfer: Fundamentals and Applications. Taylor \& Francis, New York. 